\title{
The information system of the geological and technical arrangements management on a well stock of an oil-and-gas production enterprise
}

\author{
Ivan Evsyutkin, Nikolay Markov \\ Tomsk Polytechnic University \\ Lenin's Ave., 30, Tomsk, Russia, 634050 \\ pzerag@sibmail.com, markovng@tpu.ru
}

\begin{abstract}
This article describes the problem of management processes automation of the geological and technical arrangements (GTA) on the oil-and-gas well stock. For the solution of this problem it is necessary to automate, first of all, difficult business processes of selection of candidate wells for GTA and a selection of necessary arrangements for each of relevant wells. Considering variety of the used methods for candidate wells selection for GTA and a selection of GTA, it is offered to create the information system for management of GTA on the basis of the service-oriented architecture (SOA) concept. The SOA model of information system is designed; features of program realization for the system are shown.
\end{abstract}

Key words-Information system; web-service; serviceoriented architecture; geological and technical arrangements on a well stock of the oil-and-gas production enterprise.

\section{INTRODUCTION}

Field devilment and new wells drilling on the exploited fields are labor-consuming and expensive projects therefore the majority of the oil-and-gas enterprises use intensification methods of oil production, gas and gas condensate from the existing wells as alternative approach. Thus the geological and technical arrangements (GTA) is a big class of the works on a well stock of the enterprise directed on receiving a gain of oil production, gas and gas condensate that are of special interest. Management of GTA demands considerable labor and time expenditure of qualified specialists of geological and technological enterprise departments who practically manually analyze today large volumes of diverse geological and oil-andgas field information that promotes increase in probability of mistakes emergence owing to a human factor [1]. In this regard the development of new methods, algorithms and the information systems supporting them for the automated and (or) automatic solution of GTA management problems on a well stock of the oil-and-gas production enterprise is very actual.

In this article the information systems (IS) of GTA management existing today are considered, the analysis is carried out from the point of view of systems expenses and temporary and human resources consumption, necessary at use. It is shown that the perspective direction for increase of GTA management processes automation level is creation of IS with use of the concept service-oriented architecture (SOA).
The SOA model of the developed IS is described. Features of IS program realization are shown.

\section{THE EXISTING INFORMATION SYSTEMS OF GTA MANAGEMENT}

At realization of the business processes (BP) of GTA management geological and technological departments of the oil-and-gas production enterprise need to solve the following conceptual tasks:

- Collecting basic data by results of the well stock monitoring and the operated oil-and-gas formations received from various systems of the enterprise. Specification of basic data on the basis of analysis and plans results of the of work for pressure transient test, well survey and hydrocarbon logging, and also the requirements and proposals analysis results of the production departments which consider a condition of the well stock in the activity.

- Implementation on the specified basic data of selection candidate wells procedures for carrying out GTA.

- Relevant arrangements selection for each of these candidate wells, and sometimes several.

- An assessment economic (in certain cases and technological) of the chosen type (types) of GTA efficiency for each well.

- Plans coordination of carrying out the chosen GTA with schedules of departments works of the workover (WO), appointment of WO crew for work with each well.

- Carrying out the picked-up GTA on candidate wells.

Carrying out the first five tasks is essentially impossible without automation tools, that initiate creation of GTA information management systems.

Among the above tasks the candidate wells selection of for carrying out GTA on the considered well stock and selection of the types of GTA suitable for them are the most laborconsuming and difficult automated problems. Therefore at the moment at the majority of the oil-and-gas production enterprises in Russia and at many enterprises abroad BP 
directed on the solution of these tasks are carried out in the semi-automatic mode, and only at some enterprises separate subprocesses are carried out in the high-automated mode. Methods and algorithms for the automatic mode of the such tasks solution only start developing because of specificity of basic data. Really, for each wells type (oil and gas) process of candidate wells search automation for GTA can significantly differ in connection with geological features of different types of the operated fields and because of various used sets of the parameters describing work of a well or the Well-Formation complex (Formations).

Nowadays in Russia and abroad in oil-and-gas industry for GTA management in many producing enterprises the highly specialized information systems providing the low level of automation at management of GTA are used (they use a semiautomatic operating mode of the specialists). Most often such systems deliver to the specialist and transform to the necessary data form from other information systems of the enterprise, for example, from monitoring system of a field well stock, from research of wells works plans, etc., systems allow to visualize the data transformed for the analysis at decision-making by the specialist.

In some Russian Systems of GTA management along with "hand" the automated analysis of basic data (the semiautomatic mode) is used when simple methods of the candidate wells automated selection for carrying out GTA are used. Among such methods is most often applied the geological potential method [1] allowing the specialist to calculate an expected well production of each well and, according to results of calculations, to make the decision which of them are candidates for carrying out GTA. Unfortunately, the method of geological potential is applicable only for oil wells. This method cannot be applied to oil wells with the absent values of some geological parameters because value of an indicator of geological potential of a well is computational.

In the world market of monitoring and management systems of fields development big popularity was gained by the corporate multifunctional information systems developed on the basis of software products of the known foreign companies Schlumberger, Halliburton and others [2, 3]. Such corporate systems of the oil-and-gas production enterprises include also a subsystem of management of GTA on a well stock. Each of corporate systems provides the uniform information space for the data analysis for work with diverse basic data from various subsystems of corporate system: data about current state of a well stock, the calculated results of the forecast of different geological and oil-and-gas field parameters, hydrodynamic modeling results of productive formations, etc. Use of these data, and also number of service functions (graphical data representation, three-dimensional results visualization of hydrodynamic modeling, etc.) allow the specialist to carry out in automated mode the complex analysis of data at a candidate wells selection for GTA. Unfortunately, in these systems the methods implemented as the main are not always give good results at selection of candidate wells for GTA. Moreover, such corporate systems differ in the high initial price and cost of the introduction stage. Therefore, not all Russian oil-and-gas production enterprises can buy them.

Summing up the results, it is possible to consider that the simple methods and algorithms of GTA management used today at the majority of the oil-and-gas production enterprises of Russia and abroad rely on experience of specialists of geological and technological departments, and the specialized information systems provide only partial automation of GTA management processes on a well stock.

All this indicates development topicality of inexpensive, but perspective regarding the implemented methods for the automated and (or) automatic solution of the listed above integrated tasks at GTA management on a well stock IS. Such systems have to be aimed, first of all, at the high level of automation of candidate wells selection problems for GTA and a necessary arrangements selection for each of such wells.

Considering that the system has to allow flexibly to include / exclude the program modules carrying out all variety of methods and algorithms of the candidate wells selection for GTA and methods of GTA appointment for them, it was offered to develop IS of GTA management on the basis of the concept of SOA.

\section{ARCHITECTURE OF INFORMATION SYSTEM}

Let's consider the IS architecture features of GTA management constructed on the basis of the concept of SOA. From [4] follows that SOA is a modular approach to the development of software and IS that based on the use of distributed, loosely coupled and replaceable software components (services), which are equipped with standardized interfaces for communication by standardized protocols [6]. In this case the service is standalone software component (module) with the possibility of self-description which performs a specific business task (BP, sub process, function) and provides some of the functionality by the request to IS or other services. In most cases web services are developed. Loosely coupled services provide a simple and fast adaptation of the SIS to changes in enterprise's BP. The services can work standalone or to be combined with other services to create a new composite applications (IS or SC). The services allow implementing a particular BP by their interaction on the network in a certain sequence. The service should be used according to the convention of the interface. The interfaces are used for providing the service's methods to the outside world and to organize an interaction of services. The service interface contains the description of input parameters and of the result of its work.

Another key component of SOA is a service registry. It is presented by a catalogue and includes descriptions of services (their physical location, versions and validity period; documentation for services). Due to using the service registry SOA has loosely coupled services.

The third component of SOA is an Enterprise Service Bus (ESB) [5]. The ESB is connective software for all existing (legacy) IS and SC on the enterprise and all new developed services to provide centralized and unified event-driven messaging between IS, SC and new enterprise's services. All 
settings for the processing and sending of messages are usually concentrated in a single point of ESB. It means that in case of change of any IS, SC or service connected to the bus there isn't need to reconfigure other systems and services of the SIS. The ESB is responsible for a number of classical tasks of integration of IS, SC and services:

- message routing;

- conversion of message formats and protocols;

- transaction support;

- $\quad$ providing security, auditing, logging.

Finally, another component of the SOA is Business Process Management (BPM) system. BPM system is software providing opportunities to start, coordinate, manage, administrate and debug the BP. The BP is deemed to be a sequence of logically related actions that transform input data into results or output data. In other words, BP determines a startup sequence for IS, SC and services to solve specific business task. Management of the startup sequence is called orchestration. BPM system also controls the choreography in the case of the exchange of messages between IS, SC and services.

Summarizing the brief analysis of the components of SOA and its main features, we can assume that SOA is a promising model of interaction between the SIS components (legacy IS, SC and new services). This model allows the components to communicate with each other through specified interfaces. The interfaces aren't dependent on hardware platforms, operating systems and programming languages that were used to develop components of the SIS on the enterprise. This allows components to communicate with each other by the same standard and versatile way. This aspect of using interfaces that are independent of the environment and the platform is called "loose coupling" model. This model provides greater flexibility and adaptability of the SIS in the changing requirements of the enterprise as the replacement or upgrading a component of the SIS will not affect the others.

Briefly we will stop on a selection of tools and technologies to create IS on the basis of the SOA model. For the functions description of services and messages between them are used two most often used technologies [5]:

- Web Services Description Language (WSDL) is a language for web-services and access to them; the technology of development of such services divides the description of services into the description of the interface and shell: the interface describes what must contain the request and the shell determines the data and transport protocols;

- Simple Object Access Protocol (SOAP) is a protocol for exchange structured messages that is used for description of format of received and sent messages.

The ESB should be presented by one of service buses of known software companies such as Oracle, IBM, Microsoft and etc. More full ESB functionality is presented in Oracle ESB, Open ESB and IBM Web Sphere ESB [7]. Most ESB comes with a large number of pre-configured adapters: adapters for ERP systems (SAP R/3, Oracle eBusiness Suite, 1C, MS Ax 2009), adapters for databases and etc.

The BPM-system describes the coordination of BP using the Business Process Execution Language (BPEL). The description of BP on BPEL is compiled into an executable code of the BPM system that allows to recover described BP from available IS, SC and services. In recent years a more advanced language for modeling $\mathrm{BP}$ was invented - the language BPMN (Business Process Model and Notation) [8]. BPMN specification allows to present BP as diagrams and to convert them to BPEL.

As BPM system it is possible to use widely known systems such as Bonita BPM, Orchestra (supports both languages), ELMA BPM, Bigazi BPM (support the BPMN language) and. etc. We used the Russian development ELMA BPM [7]. In fact, this BPM system is in some way the information systems development environment on the principles of SOA.

The architecture of the created IS is depicted in fig. 1.

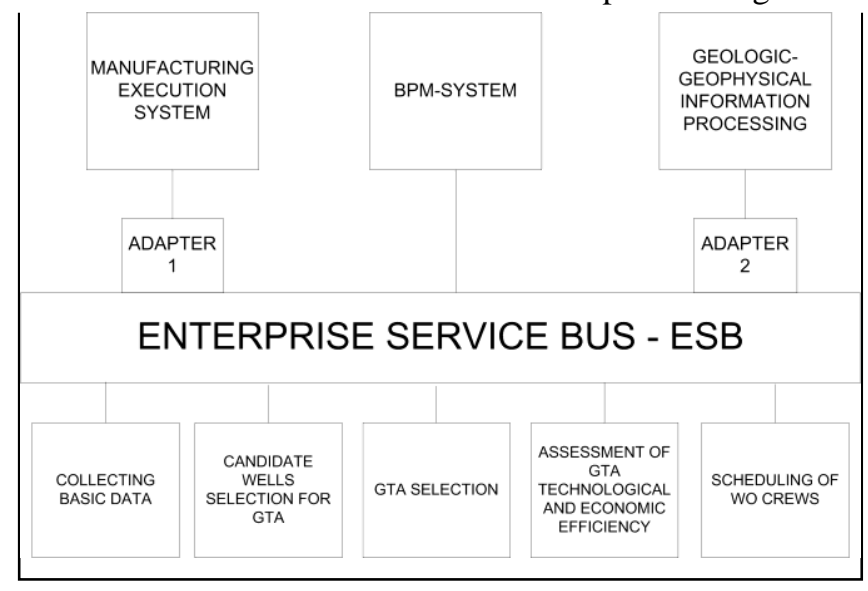

Figure 1 Architecture of IS

Let's consider its features. The main component of architecture of IS is the enterprise service bus (ESB) which functionality is implemented in the ELMA BPM environment. Through ESB the IS services interact among themselves and with information systems of the enterprise, which are external in relation to the created IS. Two systems are given as an example of external IS in fig. 1 (they along with ELMA BPM are located above the service tire). The first of these systems MES (manufacturing execution system) - is intended for operational management of the oil-and-gas production enterprise productions. Instead of it the enterprise can have simpler system of dispatch control. From such systems services obtain data about wells stops, time of their idle time etc. Other example of external IS is the system of geologicgeophysical information processing (interpretation) from which services receive, for example, values of geological parameters of productive formations. Other external IS are connected to ESB if they contain the data used by services.

External systems not necessarily have to have the standardized interfaces because they could be created not within the concept of SOA therefore for them adapters of ESB can be used. Among the services that carry out BP of GTA 
management and created within IS (in fig. 1 they are depicted below ESB), it is possible to determine the following:

- Collecting basic data. IS has to act as a superstructure in relation to the production IS which are already existing at the enterprise, IS has to provide integration with them by data. Various data bases of such systems which are already existing at the enterprise containing values of geological and oil-and-gas field parameters of wells and productive formations, proposals from production departments for carrying out GTA, annual and monthly plans of pressure transient test, well survey and hydrocarbon logging of the wells that conduct to their stops can act as the data sources brought in IS database (DB).

- $\quad$ Candidate wells selection for GTA. The main methods for selection are the automatic and automated methods of the analysis of technological parameters trends, intellectual history analysis methods of operational wells stops, and also specialized methods (calculation of geological potential for oil wells, a technique of stops planning of OJSC "Gazprom” for gas wells, etc.) [1]. It is necessary to implement in IS the algorithms of the record-keeping of plans of wells stops for researches and the wells accounting chosen earlier, but for any reasons which was reserved, etc.

- GTA selection. Depending on type of wells (oil or gas) various automatic and automated methods and algorithms of GTA selection are applied to them. IS has to include the services implementing the specified methods and to allow the specialist to choose the most effective methods in concrete situations. In other words, IS has to carry out support of decision-making by the specialist for GTA selection for each candidate well.

- Assessment of GTA technological and economic efficiency. With help of this service carry out an assessment, whether the received decision about GTA selection from the economic and technological points of view will be effective. The enterprise budget for a year is restricted and it is impossible to go beyond it usually. That is why GTA management process can be iterative (the part of candidate wells goes to a reserve, but they do not stop).

- $\quad$ Scheduling of WO crews. IS has to build the optimized plan schedule of crews work for the minimization purpose of candidate wells total idle time.

All listed services are compound as are combined from simpler services.

\section{FEATURES OF IS PROGRAM REALIZATION}

Initially the ELMA BPM development environment does not contain any information about subject domain therefore the realization essence of IS is consisted in adaptation of the given development environment to area of oil-and-gas field geology, and also in realization of all demanded functionality of IS in the form of services.
In the ELMA BPM development environment has a builtin designer allowing to design the list of necessary objects (entities) and reference books of the oil-and-gas production enterprise in the form of the FireBird database. For this purpose the development environment has not only the user interface for work with objects, but also the interface with possibility of logic of their processing with use of the SQL (Structured Query Language).

It is possible to distinguish from objects: fields, well pads, wells, productive formations, crews of WO, lists of proposals, plans of wells researches etc. Among reference books are types of GTA, standards of GTA (performance terms, GTA cost, equipment cost), units of measure, scenario conditions (rates of taxes, cost of raw materials and materials, cost of raw materials transportation) etc.

All objects include attributes which have (for the subsequent testing of IS) the real values taken from databases of one of OJSC "Gazprom" subsidiaries.

All business processes implemented in IS originally were designed in the BPMN of the ELMA BPM development environment. It also allowed to adjust the BPM-system of IS. The example of the description of BP "Candidate wells selection for GTM" is shown in this notation in fig. 2.

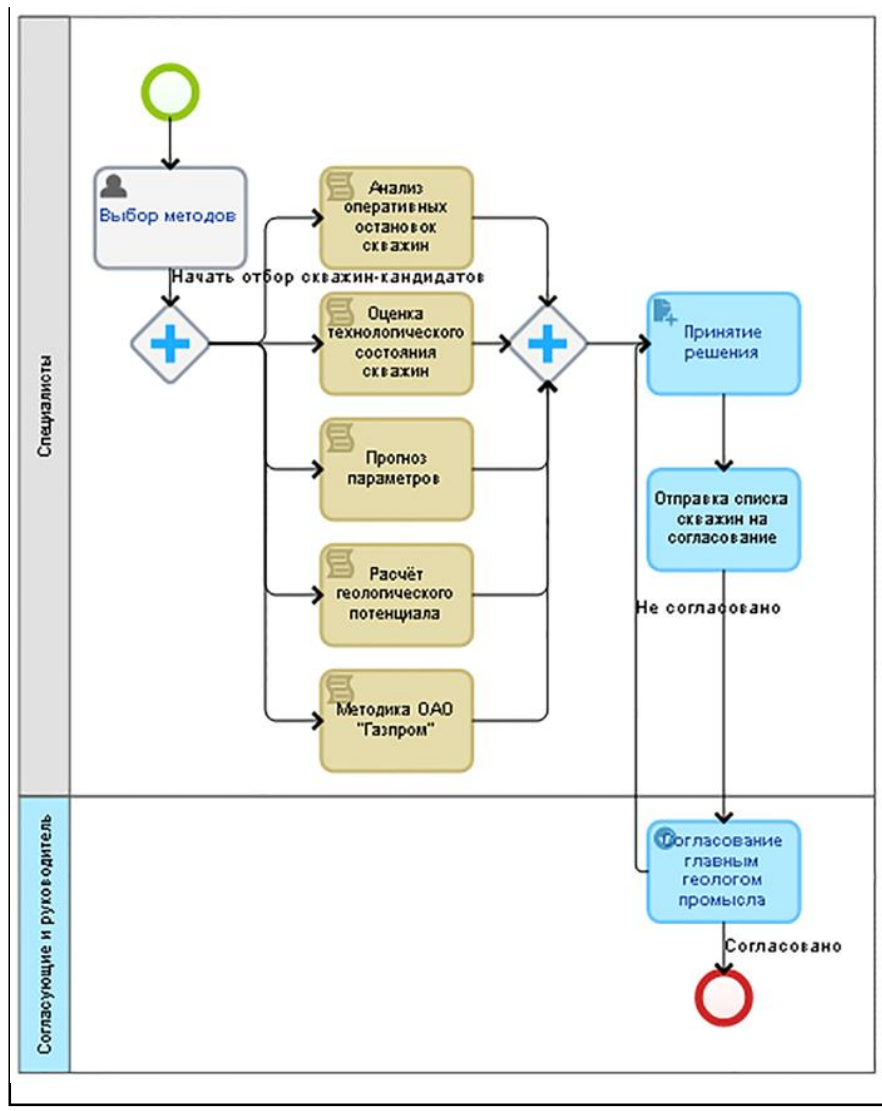

Figure 2 BPMN chart BP "Selection of candidate wells for GTM"

The chart shows that the specialist at first can choose a method of candidate wells selection for GTA, and then start it for actually selection of wells. If the result does not suit it, specialist can use other method from five methods represented 
on the chart. Each method is implemented in the form of separate simple service.

It should be noted that the BPMN language contains very full set of various elements that allows to describe any BP. BPMN includes events. Except an initial and final event, for BP can be used the timer, for example, for monthly collecting proposals for GTA from production departments. There is a possibility of branching of BP, control of responsibility zones, management of documents flow.

Subprocesses in BP can be carried out by the user in the ELMA BPM web-interface or programmatically, i.e. by a service. For programming of services the development environment in the $\mathrm{C \#}$ language where the developed webservices were also involved is built in.

Realization of the IS web-services was conducted in the C\# language. At creation of the user interfaces was applied layout ASP.NET MVC Razor. The description of services interfaces is made according to the WSDL 1.1 standard, the exchange of messages occurs under the SOAP 1.2 protocol.

During work of the oil-and-gas production enterprise often there is a need to use various forms of reports, such as plans of wells researches plans schedules of crews WO work on wells, production indicators, etc. ELMA BPM allows to adjust their formation by means of SQL on the basis of the data brought in IS DB.

For work of the specialist with IS is used the web-interface of the ELMA BPM [7] environment. The example of the adapted interface of the main page is depicted in fig. 3. The interface allows to start any $\mathrm{BP}$, to execute subprocesses (subtasks), to plan crews WO work in the built-in events calendar and to communicate with production departments.

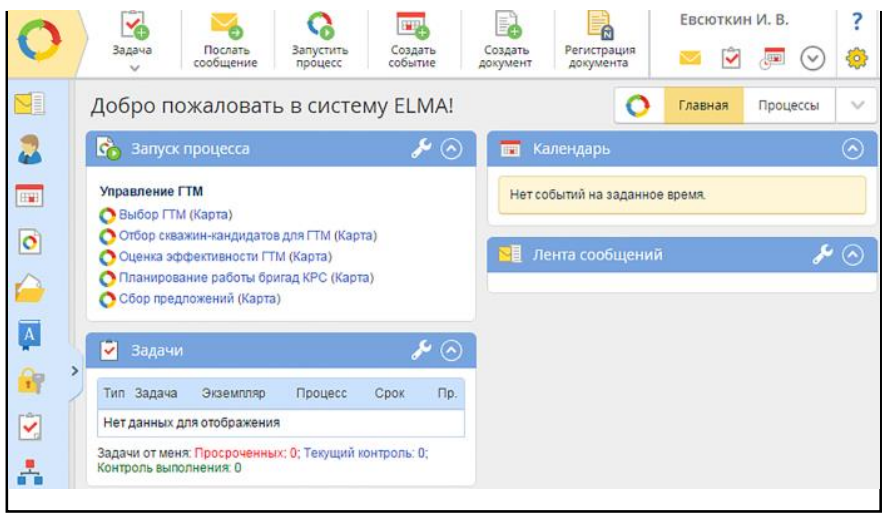

Figure 3 The main page interface of the ELMA BPM portal adapted under subject domain in IS

IS allows to work with it with in advance established set of the rights to each specialist of production departments of the enterprise, such as a chief geologist department, a chief mechanical engineer department, a chief power engineer department, an automation department, a metrology department, a production department, a communication department, a production and technological management.

\section{CONCLUSION}

The analysis of the main methods used today in oil-andgas sphere, algorithms and IS of GTA management implementing them on a well stock showed that development of the inexpensive and easily configured IS but at the same time providing the high level of automation of management processes of GTA continues to remain the actual. In this regard it is offered to create IS on the basis of the concept of SOA. The system with such architecture will allow to create easily programmatically in the form of services the main methods and algorithms of the solution of the most laborconsuming and complex challenges of candidate wells selection for GTA and appointment for them the corresponding GTA, and to the specialist working with IS will allow to select flexibly on the basis of the preliminary analysis of basic data and to apply these methods of the such tasks solution.

The SOA model of such IS is designed. Its feature is connection to the ESB not only services, but also external IS of the enterprise from which services can obtain data necessary for them.

Development of IS was carried out with use of the Russian ELMA BPM development environment which along with modeling in the BPMN language of the GTA management processes on a well stock allowed to apply in IS its BPM system and the software to develop of functions of the ESB. The IS web-services are constructed with use of C\# language, at the user interfaces creation used layout ASP.NET MVC Razor was applied.

At this moment the developed software of IS with real data use from a well stock of one of the enterprises (OJSC "Gazprom") was tested.

\section{REFERENCES}

[1] Anton Kudinov, Nikolay Markov / Problems of automation of production of the gas companies: monograph. - Tomsk: publishing house of Tomsk polytechnical university, 2012. - 247 pages.

[2] Halliburton LandMark / A Halliburton business line is the leading technology solution provider of data and analytics. - [Electronic resource]. - Access mode: https://www.landmark.solutions/, free access. - The title from the screen. - (Date of the address: 28.02.2016).

[3] Software Integrated Solutions (SIS) / Сайт компании Schlumberger. Программные продукты для геологоразведки и добычи. - [Electronic resource]. - Access mode: https://www.sis.slb.ru/, free access. - The title from the screen. - (Date of the address: 30.02.2016).

[4] Juric M. SOA approach to integrational. - Birmingham: Packt Publishing Ltd., 2007. - 366 p.

[5] WP 27. Service-Oriented Architectute in systems of production management. Official materials of MESA International // MES association - the theory and practice. - 2010. - №2 - - Pages 5-59.

[6] W. H. Utomo, T. Wellem, JATIT, 62, 364-370 (2014).

[7] BPMN - model of business processes and the notation. - [Electronic resource]. - Access mode: www.elma-bpm.ru, free access. - The title from the screen. - (Date of the address: 22.01.2016). 